\title{
ENFERMEDAD DE HODGKIN
}

\author{
Por al Dr. PROSPERO ARRIAGADA SEgUEL
}

Servicio de Pediatría del Hospital Regional de Vildivia.

Sírvame de prólogo para haçer una revisión de los conceptes actuales acerca de la naturaleza tan discutida de esta afección. de sus más modernos recursos díagnósticos y de sus Fosibilidades terapéuticas, la exposición de dos casos en ninos que en el plazo de año y medio mie ha tocado constatar y seguir de cerca en nuestro Servicio de Pediatría del Hespital Regional de Valdivia.

El primero se refiere a M. S., de 7 años de edad, procectente de Trape. No hay antecedente; familiates ni extrafamiliares de infección tuberculost. o hermanos sanos; un hermano muerto por causa no precisada. Acusós arampín a lo; 5 años de edad. Lo traen al Policlínico, poxque desde hace un año the notan un tumot del tórax que ha ido meciendo lenta iy silenciosamezte. Hai unflaquecido y está inapetente. En renlidad, aus condicioné generales no son suti:factorias, pesa $20^{\circ} \mathrm{kg} \mathrm{rs}_{\text {. }}, 37.8^{\circ}$ de temperatuta recpal: su psiquis y actividad muscular están bien. Píl pálida con elementos de sarnia. La dentadura esth́ incompleta, con varias caries dentatias. Hezy ganglios det tamaño de un poroto, libres, indoloros. por detrás del esternocleidomastoídeo a izquierda. En el tórax, por debajo de la clavicula izquierda, se ve un gran tumor con circulación venosa colateral, que a. la palpación da na supeuficie abolilonada, de consittencia blanda, indoloro. Bronquiales en ambas campos, A la auscultacion esternal alta bay estridor traqueal, maroadamente espiratorio. Hígado $y$ bazo aprecian en limites nomales. Adenopatias hasta dét tamaño de un poroto, inguinales $y$ axilares. Pessándose en una lesión de ordén quirúrgico. s: covia en interconsulta al servicio correspondiente, en donde proponen hory:talización para completar at estudio. El Mantonx al 1 x 10.000 (一) a las 24 y 48 horas. Sedimentación da $112 \mathrm{~mm}$. en 1 hora. Tórax normal a los myor. Kahn (-). La orina etidencia ligeros signos de irritanión remat. ex- 
tetiorizados por albuminuria mínima y glóbalos rojos escasos. El bemograma da onis anemia de 3.080 .000 glóbulos rojos $\mathrm{g}$ leucocitosis de 14.900 ; la fór mula de Schilling; $6 \%$ rosinófilos. $7 \%$ bacilifformes, $43 \%$ regmentados $24 \%$ linfocitos, $20 \%$ monocitos. If a cosinofilia alta nos deternina a pedir fos naevos exámenes: Reacción de Chanffard, lque resulta (-) y un parasitológico na las deposiciones, hallándose hueyos de ascaris y tricocéfias. A los 22 dias de ingreso, el estado general no sz modifica, la palidez es manifiescu. inapetencia, curvia febril de tipo intermitente y remitente (hasta 399). E1 híқ.?do se toca ahori a un través de dedo del rebotde: fl bazo igalmente salpa. Aparezon adenopatías cervicaks a derecha, hasta del semaño de una almendra. Mejotadas sus condiciones denmatológiens, se procede a tomar biopsia drel tumor toxáxico: en realidad, practica una extiroación del mismo, hue apars: formado por un enorme paqueté dé ganglios violáceos de diferentes tamaños. El anátomo patólogo envia el siguinnte diagnóstico histo patológico; nasa constituía por un pàquete de ganglios ovoideos, algunas alcanzan el vofumen de ex huevo de gallina, los más chicos como un poroto; al corte de folor zris toiizo como tocino. Al examen histológico. la estrnctura del ganglio aparece profundamente alteradia por la formación de focos celulares extensos muy polir. Orfos, entre los cuahes numetosas célnlas retículases hipertróficas multinuclealas, llamadas células de Stemtnerg. Hay esclerosis escasa y alganas zonas neŁróbióticas, Diagnóstico histopatológico: LINFOGRANULOMATOSIS MA'LIGNA GANGLIONAR DEL TDPO DE PALTAUF-STERNBERG. A los 47 dís de estada dél enfermito se anota en la observación cliniea: el estado general ha experimentado mejotia bajo la influencia de dos factores: el As (licor de Fowler) y la gangliectomía sin embargo, a esta fecha hil higado reor a 2 traveses de dedo y el bazo se tóce fácilmente: hôy grandes cadenas gangliotlares cervicales bilaterales. En la imposibilidad de poner en práctica otros recursos terapéuticos, se da de alta con indicaciones de arsenoterapia. No bemoc sabido posteriormente de él.

Diez meses más tarde, el 5 de mayo de 1942. 1lega procedanté de I.os Lagos nuestro segundo caso. Es C. T. C., de 4 años de edad, que consct. ta por tumor indoloro cervical derecho desde dos meses antes, de crecimiento Frogtesivo. En las dos íltimas semanas acontece algo semejante a izauierds. Frbril desde un comienzo, enflaquecimiento pronunciado, epistaxis en dos asin siones. Thanto sus antecedentes personales, como familiares, capecen de interé. Ai examen nos hallamos anfe un chico desnutrido, febril (39.6\% tectales), pílido, enorme aumento de volumen de las gaoglios del cuello, regiones patotistas, supra e infraclaviculares y de la nuca: los ganglios se palpan duros, lisos. libres, poto desplazables sobre los planos profundos. La piel que recubre dichas tumoraciones presenta pequeñaj vesiculas, que se interpretan como da éxtasis linfático; circulación venosa colateral acentuada. Hay ecuplas raquíticas torácicas. Eil bazo está daro a dos trapeses de čedos del reborde costal; li hígado está aumentado en uno $y$ medio través. En las regiones axilares se tocan ade1:opatias del tamañio de un poroto. La reacción de Mantoux e; (-) a las 24 
y 48 hons: reacciones serológicas igralmente regativas. La radioscopía de tó$\mathrm{r} x$ pone en eviáencia una adenopatía hijiar jzquierda. Ia orina deja sedimento abundante constituído por erato de amonio, indicios de albúmina, glucesa 7,8 grs. $0 / 00$. El hemognama: glóbulos rojos, 4.040 .000 con norro. Elastos $1 \%$; la fónma blanca da una reacción lencémica aleucémica: glóbulos biancos, 20,200; mielocitos, 4; juveniles, 1; baciliformes, 24; segmentados, 50; linfocitos, $\vec{r}$; monocitos. '5. Se toma biopsia de uno de los gauglios del: arallo; el informe histológico dice: en el ganglio enviado se observa aué at tijido linfó́deo bi sido sustituido por tejido de granulación, en el que ven crilulas reticulanes muy aumentadas, algunas células de Sternberg. linfocitos. vesos $y$ un retículo fibroso difuso; en general, la estructura del ganglio ba vido completamente modificadu. Diıgnóstico histopatológico: ENFERMEDAT DF. HODGKIN O ENEERMEDAD DE PALTAUF-STERABERG inr Aliaga). La evolución que siguió este caso es la que a continuación se señala: febriculas en las tardes: al $5 .^{\circ}$ dia, deposiciones finidas fdecuentes: al $8 .^{\circ}$ día cuarete tos, edema discreto de manos y antebrazos. A los 12 días, estridot, dasnea. tos de carácter irritativo, gran decaimiento. A los 15 dias, se generdlizan los edemas: las deposiciones están semiformadas: el bazo se toxa $x$ don v medio traveses $y$ el higado a tres; bronquiales ambos campos $y$ forn brosconeumónico de la base derecha. Fallece al dia siguiente. A la autorisia * hatla gran anmento de volumen te todos los ganglios del árbol respitatorio. incluso intraparengaimatosos palmonares a izquierda, en la región yuxtahilizr: fncos bronconeumónicos confluentes de ambos pulmones: el típico bazo de pórfito: Edenopatias hiliares viscerales $v$ del mesenterio hasta del tamaño de una castaña: las Jesiones ganglionares ya descritas al examen externo.

El nombre de "Enfermedad de Hodgkin" parece menos objetable de todos los títulos aplicados a esta afección, primeramente descrita por Hodgkin en 1832. Vayan sólo a título informativo los siguientes: Pseudoleucemia: Adenia: Linfadenia: Anemia linfática; Linfogranuloma; Linfoblastoma; Linfoma maligno; Reticulosis aleucémica.

Su aparición en la infancia no es rara. Hay incluso trabajos recientes que la catalogan como una afección relativamente frecuente en este periodo de la vida. Con mayor frecuencia se constata su iniciación en la tercera infancia: pero aun puede hacerlo en los primeros meses de la vida, siendo el caso más precoz el de un lactante de 4 meses de edad, recientemente publicado en el Journal of Pediatrics de agosto del presente año. Es más común en hombres que en mujeres y suele tener un carácter familiar, habiéndose citado casos de gemelos que presentarón simultáneamente la afección. Es interesante la observación de mujeres embarazadas con enfer- 
medad de Hodgkin que dieron a luz hijos sanos, presentándose a breve plazo la afección en el lactante.

Aun se desconoce su causa. La gran controversia ba girado siempre acerca de si el granuloma es una enfermedad de carácter inflamatorio o si se trata de una neoplasia. Es de todos conocido la importancia que se daba a la tuberculosis como causa posible; peto opiniones autorizadas sostienen gue cuando la tuberculosis está presente, debe ser considerada como una infección secundaria. Los hay que sostienen la naturaleza infecciosa de la enfermedad, basándose en el curso clínico. el carácter de los cambios anátomo-patológicos y 1 a producción de pequeñas epidemias; sin embargo, en la incculación experimental en animales, han fracasado sistemáti. camente. La tendencia general moderna es a considerar esto cuadro. al menos en sus períodos primeros o intermediarios. como un proceso inflamatorio, probablemente causado por varios estímulos que actúan sobre los ganglios linfáticos, priri cipalmente estímulos de naturaleza tuberculosa. Una vez esrablecida esta reacción inflamatoria, que por su intensidad debiera llamarse hiperreacción, el proceso ganglionar continúa desarrollándose indepen ¿entementr d 1 est mulo primarin v en ciertos casos con caracteres neoplásicos (el sarcoma de Hodgkin): A la luz de estos nuevos conceptos, pierde toto interés la búsqueda de un factor etiológico determinado par: precisar cuáles son las circunstancias que motivan este tipn tan especial de reacción ganglionar en ciertos individuos.

Desde el punto de vista histopatológico, la caracteristica de la enfermedad de Hodgkin consiste en una biperplasis del tejido linfático en donds quiera tive se halle. Los ganglios muestran prolifaración $d$ z 7. células atículozndotelidles, células eosinófilas. células linfocitarias y las peculiaras células gigantes de Sternberg.

El cuadto clínico es generalmente de comienzo insidio so. siendo el primer sintoma el aumento de volumen de al guno de los grupos ganglionares, con mayor frecuencia cer vicales y a izquierda. Desmejoran las condiciones generalo. inapetencia, fiebre ce tipo irregular en muchos casos. General. mente pasan meses o años antes que la enfermedad hage nuevos progresos. El hígado y el bazo crecen. Apare anemia, aunque de grado discreto. Las modificaciones de ? Sórmula blanca no son constantes: leucocitosis polimorfort?. clear: leucopenias; a veces, eosinofilia. Algunos hablan de aumento del número de plaquetas, afirmación que otros niegan. Los fenómencs compransivos determinados por lis masas tumorales gangitonarcs llevan a la producción ce los 
más variados cuadros clínicos: edemas, dolores, ictericia. tras tornos urinarios, sintomas nerviosos, taquicardia paroxística: tos. disnea, disfagia, etc., entre otros síntomas, a veces prosentes, se pueden citar las albuminurias, derrame pleural o pericárdico, pigmentación y descamación de la piel, nódulos cutáneos, prurito, urticaria, dolor a lo largo de los huesos, etc. A medida que la enfermedad avanza, los ganglios adquieten mayor volumen, la esplenomegalia se acentúa y se instala caquexia progresiva. Mueren por caquexia, degeneración amiloídea. bronconeumonía o como consecuencia de $10 s$ fenómenos compresivos.

La duración media de la enfermedad es de 2 años. Al lado de casos de rápida evolución hay otros que duran años $v$ son muy influenciados por el tratamiento. No son raras las remisiones, con mejoría de todos los síntomas, incluso la remisión de las tumoraciones ganglionares.

Ciagrı́stico diferencial. -- La adenitis cuborculosa va al reblandecimiento y supuración, lo que nunca acontece en 13 enfermedad de Hodgkin; además, corrientemente es locaiizada: sin embargo, en un comienzo el diagnóstico diferencial es bastante dificil, por lo cual la biopsia suele ser indispensible para catalogar un cuadro dudoso. Puede ser de alguna utilidad la negatividad del Mantoux en el Hodgkin: pero debe tenerse presente que aun habiendo asociación con adenitis tuberculosa, puede resultar negativa la prueba tuba.culínica, a causa de la anergia existente. El hemograma permite excluir la leucemia linfática. Se han citado casos de aso ciación de Hodgkin con leucemia mieloídea. El linfosarcom? s? caracteriza por la invasión de los tejidos periganglionates, lo que raramente ocurre en Hodgkin: tampoco hay esple nomegalia en la primera afección. Puede coexistir el linte sarcoma y la enfermedad de Hodgkin en el mismo paciente

Ya hemos hecho resaltar la importancia del diagnóstico histcpatológico, que es decisivo. Velasco Montes llama I atención hacia las ventajas de la adenopunción sobre la biopsia ganglionar, a la que considera no exenta de riesgos; n! plea regularmente la adenopunción en los casos dudosos. sustituyéndola a la biopsia: pero es neceszria una rlan experioncia hematológica para una correcta interpretación de los resultados. La punción de la medula esternal como recurso diagnóstico no es práctica por sus dificultades técnicas v por lo demás, sólo se compromete en la mitad de los casos. La función esplénica, a la que hay que recurrir en ciertos $c a-$ sos, da células de Sternberg tipicas. En los últimos años 
describe el test de Gordon, de orden biológico y que consiste en un sindrome meningoencefálico determinado en el conejo por la inyección intra-cerebral de caldo de suspensión de ganglios linfáticos alterados procedentes de un enfermo de Hodgkín (parálisis espástica, incoordinación, ataxia, retracción de la cabieza, pérdida del peso). Esta reacción se da positiva para el $73,9 \%$ a $76.2 \%$ de los casos; es indudable que en forma alguna remplaza al estudio histopatológici. siendo más bien una ayuda a éste para la resolución de los casos difíciles. Referente al mecanismo en juego en el test de Gordon, se piensa que lo más posible es que se trate de un agente de naturaleza fermentativa proteolítica, ya quc cs difícil aceptar la existencia de un virus o de una neurotoxina.

La radioterapia sigue siendo el método de elección para el tratamiento. Su objetivo es obtener una remisión tan larga como sea posible, procurando al paciente ana sensación de salud perfecta. Se hacen aplicaciones diarias sobre las diversas regiones comprometidas en orden sucesivo, hasta la destrucción total de los focos granulomatosos superficiales y profundos, evitando toda repercusión desfavorable sobre el estado general y cuadro sanguíneo. Se repite el mismo tratamiento en caso de recidivas. Conviene tenet presente que los trata. mientos irradiatorios con fines preventivos de las recidivas son enteramente ineficaces y aun perjudiciales. Coadyuvan al tratamiento radioterápico las transfusiones sanguíneas, lo. preparados ferruginosos y un régimen dietético de sobrezli. mentación como para tuberculosos.

Se han hecho ensayos terapéuticos de orden biologici. que, puede decirse, aun no han salido del campo experimental. Cítanse casos que se han beneficiaco con vacunas oreparadas con suspensiones de ganglios enfermos, como asimismo otros tratados con suero de enfermos previamente sometidos a ra-. dioterapia.

Agreguemos finalmentie que la extirpación quirúrgica de los ganglios linfáticos acarrea el peligro de una rocidiva posterior. generalmente más grave que la enfermedad inicial. 


\section{Bibliografía.}

RADIOTHERAPY IN HODGKIN'S DISEASE. THE JOURNAL OF THE AMERICAN MEDICAL ASSOCIATION. - Vol. 112, N.? 18, pág 1.863. 1939.

HEMATOLOGIC DIAGNOSIS OF HODGKIN'S DISEASE. THE JOUT. NAL OF THE AMERICAN MEDICAL ASSOCIATION. - Vol, 112. pág. $2,374, \mathrm{~N} .922,1929$.

I:TIOLOGY OF HODGKIN'S DISEASE. THE JOURNAL OF THE AME

RICAN MEDICAI ASSOCIATION. - Vol. 112, N.9 23, pág. 2.48? 1939 .

CONFERENCES OF THERAPY. THE JOURNAL OF THE AMERICAR

MEDICAL ASSOCIATION. - VoI. N. 115, N."4, pág. 298, 1940

I.LNPHOGRANULOMATOSIS MALIGNA IN OHTLDHOOD. THE JOUR

NAL OF THE AMERICAN MEDICAL ASSOCIATION. - VoI. 112 N. 14. pág. 1,259. 1942.

KELIABILITY AND SIGNIFICANCE OF THE GORDON TEST IN HODGKIN'S DISEASE. ARCHIVES OF PATHOLOGY. - Vol. 3: N.? 1. pág. 1. 1941.

THE DISEASES OF INFANTS AND CHILDREN. - P'́g. 1,047, 1938 By Crozer and Mitcbell.

HODGKIN DISEASE.IN AN INFANT. THE JOURNAL OF PETHA TRICS. - Vol. 21, N.92. pág. 238. 